\title{
Carbon nanocones/disks as new coating for solid-phase microextraction
}

\author{
Juan Manuel Jiménez-Soto, Soledad Cárdenas, Miguel Valcárcel \\ Department of Analytical Chemistry \\ University of Córdoba \\ Marie Curie Building (Annex), Campus de Rabanales, E-14071 Córdoba, Spain \\ qa1meobj@uco.es
}

The extraordinary properties of the nanostructured materials have boosted the development of new analytical tools which exploit such outstanding characteristics. Among them, the enhanced sorbent capacity of the nanomaterials in comparison with commercial sorbents has been used for the development of more efficient analytical processes in which the solid is presented in different configurations: cartridges, miniaturized columns/capillaries or immobilized in capillaries/wires.

Carbon nanocones were accidentally discovered in 1994 and firstly synthesized in 1997. The solid materials consisted of $20 \mathrm{wt} \%$ carbon nanocones, $70 \mathrm{wt} \%$ carbon disks and 10 $\mathrm{wt} \%$ amorphous carbon. Geometrically, they are rolled-up graphene sheets with one or more pentagonal rings defining a conical apex, as it has been seen through the transmission electron microscopic micrographs. These pentagonal rings provide a unique electronic distribution which clearly differences conical carbon nanostructures from carbon nanotubes and fullerenes. The analytical applications of carbon nanocones are still quite limited, however, and fall in the field of solid-phase extraction, in which surpassed carbon nanotubes thanks to their lower aggregation tendency.

In this communication, we present the evaluation of carbon nanocones/disks as new coating for solid-phase microextraction. The nanostructures were immobilized in a stainless steel needle by means of an organic binder. The fiber coating obtained was ca. $50 \mu \mathrm{m}$ of thickness and $35 \mathrm{~mm}$ in length. The evaluation of the sorbent capacity was carried out through the determination of volatile compound in water samples following the headspace sampling modality of solid-phase microextraction (HS-SPME). All the variables affecting the extraction process were optimized to obtain the maximal analytical signal. The analytes were thermally desorbed from the fiber prior to their determination by gas chromatography - mass spectrometry. The limits of detection were in the low nanogram per milliliter level and the reproducibility was better than 10 $\%$ using different fibers. The optimized procedure was applied to the determination of the selected volatile compounds in river, swimming pool, tap and well water collected from different locations. The recovery values obtained demonstrated the usefulness of the carbon nanocones/disks as sorbent material in solid-phase microextraction. 